\title{
Neutron Scattering from Magnetic Excitations in $\mathrm{Bi}_{2} \mathrm{Sr}_{2} \mathrm{CaCu}_{2} \mathrm{O}_{8+\delta}$
}

\author{
H.F. Fong ${ }^{(1)}$, P. Bourges ${ }^{(2)}$, Y. Sidis ${ }^{(2)}$, L.P. Regnault ${ }^{(3)}$, A. Ivanov ${ }^{(4)}$, \\ G.D. $\mathrm{Gu}^{(5)}$, N. Koshizuka ${ }^{(6)}$, and B. Keimer ${ }^{(1,7)}$
}

1 Department of Physics, Princeton University, Princeton, NJ 08544, USA

2 Laboratoire Léon Brillouin, CEA-CNRS, CE Saclay, 91191 Gif sur Yvette, France

3 CEA Grenoble, Département de Recherche Fondamentale sur la matière Condensée, 38054 Grenoble cedex 9, France

4 Institut Laue-Langevin, 156X, 38042 Grenoble Cedex 9, France

5 Department of Advanced Electronic Materials, School of Physics, University of New South Wales, Sydney 2052, Australia

6 SRL/ISTEC, 10-13, Shinonome 1-chome, Koto-ku, Tokyo 135, Japan

7 Max-Planck-Institut für Festkörperforschung, 70569 Stuttgart, Germany 
Many physical properties of the copper oxide high temperature superconductors appear to defy the conventional (one-electron) theory of metals, and the development of new theories incorporating strong electron correlations is currently at the forefront of condensed matter physics. Inelastic neutron scattering provides incisive information about collective magnetic excitations that is required to guide this effort. Such measurements have thus far proven possible for only two of the many families of high temperature superconductors, $\mathrm{La}_{2-\mathrm{x}} \mathrm{Sr}_{\mathrm{x}} \mathrm{CuO}_{4}$ [1] and $\mathrm{YBa}_{2} \mathrm{Cu}_{3} \mathrm{O}_{6+\mathrm{x}}$ [2][10], because suitably large single crystals of other copper oxide compounds could not be grown. While the magnetic spectra of both materials bear certain similarities, there are also pronounced differences that have hampered a unified description of the spin dynamics in the copper oxides. In particular, a sharp resonant spin excitation dominates the spectrum in the superconducting state of $\mathrm{YBa}_{2} \mathrm{Cu}_{3} \mathrm{O}_{6+\mathrm{x}}$ [3]- 10], but is not found in $\mathrm{La}_{2-\mathrm{x}} \mathrm{Sr}_{\mathrm{x}} \mathrm{CuO}_{4}$ [i]. Here we report the discovery of a magnetic resonance peak in the superconducting state of a different copper oxide system, $\mathrm{Bi}_{2} \mathrm{Sr}_{2} \mathrm{CaCu}_{2} \mathrm{O}_{8+\delta}$, made possible by the synthesis of a sizable single crystal and modern neutron optics. This provides evidence of the generality of this unusual phenomenon among the copper oxides and greatly extends the empirical basis for its theoretical description.

The magnetic resonance peak observed in optimally doped $\mathrm{YBa}_{2} \mathrm{Cu}_{3} \mathrm{O}_{6+\mathrm{x}}$ (superconducting transition temperature $\mathrm{T}_{\mathrm{c}}=93 \mathrm{~K}$ ) is a sharp collective mode that occurs at an energy of $40 \mathrm{meV}$ and two-dimensional wave vector $\mathbf{Q}_{\|}=(\pi / a, \pi / a)$, where $a$ is the nearest-neighbor $\mathrm{Cu}-\mathrm{Cu}$ distance [3]-[0]. Its intensity decreases continuously with increasing temperature and vanishes above $\mathrm{T}_{\mathrm{c}}$. In underdoped $\mathrm{YBa}_{2} \mathrm{Cu}_{3} \mathrm{O}_{6+\mathrm{x}}$, the mode energy decreases monotonically with decreasing hole concentration [8]- 10], while the superconducting energy gap is believed to remain approximately constant. Such a collective mode has not been observed in conventional superconductors. Several microscopic mechanisms have been proposed, ranging from band structure singularities [11]-[13] to antiferromagnetic spin fluctuations [14]-[19] and interlayer pair tunneling [20]. In all of these models, the interactions that give rise to the resonant mode are the same that cause pairing of the electrons in the superconducting state, so that this phenomenon provides a direct clue to the mechanism of high temperature superconductivity. According to another recent theory, the resonance mode is a manifestation of a new symmetry group linking antiferromagnetism and superconductivity [21, 22]. 
In the framework of this model, the spectral weight of the resonance peak is in fact directly proportional to the superconducting condensation energy 23, 24].

A very sensitive test of these disparate models is whether they are capable of providing detailed descriptions of both the inelastic neutron scattering (INS) results and those of angle-resolved photoemission spectroscopy (ARPES), a complementary momentum-resolved experimental technique that primarily probes single-electron excitations. By far the best ARPES data have been obtained on $\mathrm{Bi}_{2} \mathrm{Sr}_{2} \mathrm{CaCu}_{2} \mathrm{O}_{8+\delta}$ [25, 26], a material for which no INS data have been available. This situation, which has precluded a direct, quantitative comparison of the results of both techniques, is remedied by the present study.

A look at the chemical bonding in the copper oxides reveals the origin of these experimental difficulties. In $\mathrm{Bi}_{2} \mathrm{Sr}_{2} \mathrm{CaCu}_{2} \mathrm{O}_{8+\delta}$, for instance, the $\mathrm{CuO}_{2}$ planes are separated by several charge reservoir layers that interact only weakly in the $\hat{c}$-direction (perpendicular to the layers). $\mathrm{Bi}_{2} \mathrm{Sr}_{2} \mathrm{CaCu}_{2} \mathrm{O}_{8+\delta}$ therefore cleaves easily along the $\mathrm{CuO}_{2}$ layers, which greatly facilitates surfacesensitive techniques such as ARPES. This same property is responsible for the lack of INS data on this compound. In order to take advantage of the momentum resolution of INS, the signal from a large volume of material has to be coherently superposed, which is only possible if a single crystal is available. However, because of the weak bonding along $\hat{c}, \mathrm{Bi}_{2} \mathrm{Sr}_{2} \mathrm{CaCu}_{2} \mathrm{O}_{8+\delta}$ crystals typically grow as thin plates with volumes much too small for INS. The present study was performed on a comparatively large single crystal of volume $10 \times 5 \times 1.2 \mathrm{~mm}^{3}$ and mosaicity $\sim 1^{\circ}$, synthesized by the floating zone technique as described previously [27]. Its superconducting transition temperature (measured by SQUID magnetometry) is $\mathrm{T}_{\mathrm{c}}=91 \mathrm{~K}$, with a width of $2 \mathrm{~K}$.

The crystal was mounted in an aluminum can filled with helium exchange gas and attached to the cold finger of a closed cycle helium refrigerator. The INS experiments were performed at the spectrometers $2 \mathrm{~T}$ at the Laboratoire Léon Brillouin, Saclay, France, and IN8 at the Institut Laue-Langevin in Grenoble, France. Both spectrometers use neutron optics that focus the beam both parallel and perpendicular to the scattering plane, with a resulting gain in neutron flux on the sample that proved crucial for this experiment. Identical configurations $[\mathrm{Cu}(111)$ monochromator, $\mathrm{PG}(002)$ analyser, $35 \mathrm{meV}$ fixed final energy] were used at both spectrometers, so that the data can be directly compared after correcting for the different neutron fluxes at both reactors. The inelastic cross section was put on an absolute scale by a calibration against the incoherent scattering of vanadium. The comparison with $\mathrm{YBa}_{2} \mathrm{Cu}_{3} \mathrm{O}_{7}$ (below) is also facilitated by the availability of data taken 
on the same spectrometer under the same experimental conditions [6].

As our $\mathrm{Bi}_{2} \mathrm{Sr}_{2} \mathrm{CaCu}_{2} \mathrm{O}_{8+\delta}$ crystal has a similar superconducting transition temperature as optimally doped $\mathrm{YBa}_{2} \mathrm{Cu}_{3} \mathrm{O}_{6+\mathrm{x}}$, we began our search for the resonance mode in an energy range around $40 \mathrm{meV}$. Fig. 1a shows scans of the neutron intensity as a function of the in-plane wave vector $\mathbf{Q}_{\|}$at a constant excitation energy of $43 \mathrm{meV}$. A featureless background, mostly due to multiphonon processes commonly observed in materials with large unit cells, is present at all temperatures. While no signal can be identified above this flat background at $\mathrm{T}=100 \mathrm{~K}$, a pronounced peak centered at $\mathbf{Q}_{\|}=(\pi / a, \pi / a)$ appears at lower temperature. The peak amplitude measured at symmetryequivalent points in reciprocal space decreases with increasing wave vector, providing reassurance that the peak is of magnetic origin, and it compares well to the magnetic form factor of copper measured in antiferromagnetically long-range ordered copper oxides.

Indeed, this behavior is one of the hallmarks of the magnetic resonance peak in the superconducting state of $\mathrm{YBa}_{2} \mathrm{Cu}_{3} \mathrm{O}_{6+\mathrm{x}}$. Another characteristic feature of the resonance peak is revealed by a scan of the wave vector $\mathbf{Q}_{\perp}$ perpendicular to the $\mathrm{CuO}_{2}$ planes. As shown in Fig. 1b, the additional low temperature intensity is sinusoidally modulated as a function of $\mathbf{Q}_{\perp}$, with a periodicity equal to the inverse of the distance of two adjacent $\mathrm{CuO}_{2}$ planes. (Because of kinematical restrictions at low $Q$ and phonon contamination at high $Q$, only one of the maxima of the sinusoid is accessible.) The modulation is a consequence of strong magnetic interactions between adjacent layers, whose microscopic nature has been the focus of much debate in the context of the magnetic resonance peak [15. 21].

We now proceed to demonstrate the presence of the remaining signatures of the magnetic resonance peak in the $\mathrm{Bi}_{2} \mathrm{Sr}_{2} \mathrm{CaCu}_{2} \mathrm{O}_{8+\delta}$ data. Fig. 2 shows a scan of the additional low temperature intensity as a function of the excitation energy, with the wave vector fixed at the maximum in both $\mathbf{Q}_{\|}$and $\mathbf{Q}_{\perp}$. The intensity is peaked around an energy of $\sim 43 \mathrm{meV}$, with a width of $\sim 10-15 \mathrm{meV}$. Finally, Fig. 3 shows the temperature dependence of the peak amplitude which vanishes above the superconducting transition temperature, to within the experimental uncertainty.

The magnetic excitation spectra of $\mathrm{Bi}_{2} \mathrm{Sr}_{2} \mathrm{CaCu}_{2} \mathrm{O}_{8+\delta}$ and $\mathrm{YBa}_{2} \mathrm{Cu}_{3} \mathrm{O}_{7}$ thus exhibit an unmistakable similarity. In the superconducting state, the magnetic intensity is sharply concentrated around a single point in energy and wave vector; in the normal state, the intensity is either too broad or too weak to be observable above background. There is also no indication of magnetic intensity above background level at other energies or wave vectors. In particular, an extensive search for magnetic excitations at $10 \mathrm{meV}$, an energy below the onset of optical phonon scattering where magnetic exci- 
tations, if present, should be relatively easy to observe, has thus far been fruitless in $\mathrm{Bi}_{2} \mathrm{Sr}_{2} \mathrm{CaCu}_{2} \mathrm{O}_{8+\delta}$. In both $\mathrm{YBa}_{2} \mathrm{Cu}_{3} \mathrm{O}_{7}$ and $\mathrm{Bi}_{2} \mathrm{Sr}_{2} \mathrm{CaCu}_{2} \mathrm{O}_{8+\delta}$, the resonance peak is therefore by far the most prominent feature in the magnetic excitation spectrum.

A further important comparison between both compounds is made possible by a calibration of the absolute neutron cross section against a vanadium standard. Expressed in the same units as in Ref. 28], the energyintegrated spectral weight of the resonance peak at $\mathbf{Q}_{\|}=(\pi / a, \pi / a)$ is $\int d(\hbar \omega) \chi^{\prime \prime}(\mathbf{Q}, \omega)=(1.9 \pm 1) \mu_{B}^{2}$, where $\chi^{\prime \prime}$ is the imaginary part of the dynamical spin susceptibility. (The large error bar is mostly due to the uncertainty in the energy width, Fig. 2). To within the experimental error, this is identical to $1.6 \mu_{\mathrm{B}}^{2}$ determined in $\mathrm{YBa}_{2} \mathrm{Cu}_{3} \mathrm{O}_{7}$ [7, 29]. Surprisingly, the width of the resonance in $\mathbf{Q}_{\|}$is much larger in $\mathrm{Bi}_{2} \mathrm{Sr}_{2} \mathrm{CaCu}_{2} \mathrm{O}_{8+\delta}\left(0.52 \AA^{-1}\right.$ full width at half maximum) than in $\mathrm{YBa}_{2} \mathrm{Cu}_{3} \mathrm{O}_{7}\left(0.25 \AA^{-1}\right)$. If averaged over the two-dimensional Brillouin zone, in addition to integrating over $\hbar \omega$, the resonance spectral weight is therefore clearly larger in $\mathrm{Bi}_{2} \mathrm{Sr}_{2} \mathrm{CaCu}_{2} \mathrm{O}_{8+\delta}$ $\left(\int d \mathbf{Q}_{\|} d(\hbar \omega) \chi^{\prime \prime}(\mathbf{Q}, \omega) / \int d \mathbf{Q}_{\|}=0.23 \mu_{B}^{2}\right)$ than in $\mathrm{YBa}_{2} \mathrm{Cu}_{3} \mathrm{O}_{7}\left(0.043 \mu_{B}^{2}\right.$ [29]).

Such quantitative comparisons between different materials are indispensable for a microscopic, quantitative description of the origin of the magnetic resonance peak. In the framework of the models proposed for the resonance peak, it should now be possible to relate the different $\mathbf{Q}$-widths measured in $\mathrm{YBa}_{2} \mathrm{Cu}_{3} \mathrm{O}_{7}$ and $\mathrm{Bi}_{2} \mathrm{Sr}_{2} \mathrm{CaCu}_{2} \mathrm{O}_{8+\delta}$ to their respective Fermi surfaces as measured by ARPES. (The difference in the energy widths may be more difficult to interpret, as recent experiments on Zn-substituted $\mathrm{YBa}_{2} \mathrm{Cu}_{3} \mathrm{O}_{7}$ indicate that it is very sensitive to disorder [29]. Incidentally, this sensitivity may account for the absence of the resonance peak in $\mathrm{La}_{2-\mathrm{x}} \mathrm{Sr}_{\mathrm{x}} \mathrm{CuO}_{4}$, where disorder due to Sr donors is significant. The Q-width, on the other hand, is not strongly affected by impurities.) There have also been attempts to relate more subtle features of the ARPES spectra, such as the well-known "dip" in the spectral function, to the collective spin dynamics 30, 31. These features are only clearly apparent in the ARPES work on $\mathrm{Bi}_{2} \mathrm{Sr}_{2} \mathrm{CaCu}_{2} \mathrm{O}_{8+\delta}$, and our INS data will now allow such arguments to be refined and put on a quantitative footing. The same applies to recent models that propose a direct relation between the spectral weight of the resonance peak and the superconducting condensation energy [23, 24].

Our study opens the way for a variety of further neutron experiments, in particular in the overdoped regime that is easily accessible in $\mathrm{Bi}_{2} \mathrm{Sr}_{2} \mathrm{CaCu}_{2} \mathrm{O}_{8+\delta}$ over a wide range of hole concentrations. It also leaves open questions that can only be answered by neutron scattering work on other families of high temperature superconductors. For instance, as both $\mathrm{YBa}_{2} \mathrm{Cu}_{3} \mathrm{O}_{7}$ and 
$\mathrm{Bi}_{2} \mathrm{Sr}_{2} \mathrm{CaCu}_{2} \mathrm{O}_{8+\delta}$ are bilayer materials, the present study does not provide further insight into the role of interlayer interactions in the resonance peak. Most importantly, observation of the resonance peak in $\mathrm{Bi}_{2} \mathrm{Sr}_{2} \mathrm{CaCu}_{2} \mathrm{O}_{8+\delta}$ rules out the possibility that this phenomenon is due to a conspiracy of structural or chemical parameters peculiar to $\mathrm{YBa}_{2} \mathrm{Cu}_{3} \mathrm{O}_{7}$. Rather, it is an intrinsic feature of the copper oxides whose explanation must be an integral part of any theory of high temperature superconductivity.

Acknowledgments. We acknowledge useful discussions with A.H. Moudden. The work at Princeton University was supported by the MRSEC program of the National Science Foundation, and by the Packard and Sloan Foundations. 


\section{References}

[1] Kastner, M.A,, Birgeneau, R.J., Shirane, G. \& Endoh, Y. Magnetic, transport, and optical properties of monolayer copper oxides, Rev. Mod. Phys. 70, 897-928 (1998).

[2] Tranquada, J.M., Shirane, G., Keimer, B., Shamoto, S. \& Sato, M. Neutron Scattering Study of Magnetic Excitations in $\mathrm{YBa}_{2} \mathrm{Cu}_{3} \mathrm{O}_{6+\mathrm{x}}$, Phys. Rev. B40, 4503-4516 (1989).

[3] Rossat-Mignod, J. et al. Neutron Scattering Study of the $\mathrm{YBa}_{2} \mathrm{Cu}_{3} \mathrm{O}_{6+\mathrm{x}}$ System, Physica C185-189, 86-92 (1991).

[4] Mook, H.A, Yethiraj, M., Aeppli, G., Mason, T.E. \& Armstrong, T. Polarized Neutron Determination of the Magnetic Excitations in $\mathrm{YBa}_{2} \mathrm{Cu}_{3} \mathrm{O}_{7}$, Phys. Rev. Lett. 70, 3490-3493 (1993).

[5] Fong, H.F. et al. Phonon and Magnetic Neutron Scattering at $41 \mathrm{meV}$ in $\mathrm{YBa}_{2} \mathrm{Cu}_{3} \mathrm{O}_{7}$, Phys. Rev. Lett. 75, 316-319 (1995).

[6] Bourges, P., Regnault, L.P., Sidis, Y., \& Vettier, C. Inelastic-neutronscattering study of antiferromagnetic fluctuations in $\mathrm{YBa}_{2} \mathrm{Cu}_{3} \mathrm{O}_{6.97}$, Phys. Rev. B53, 876-885 (1996).

[7] Fong, H.F., Keimer, B., Reznik, D., Dogan, F. \& Aksay, I.A. Polarized and unpolarized neutron-scattering study of the dynamical spin susceptibility of $\mathrm{YBa}_{2} \mathrm{Cu}_{3} \mathrm{O}_{7}$, Phys. Rev. B54, 6708-6720 (1996).

[8] Fong, H.F., Keimer, B., Milius, D.L. \& Aksay, I.A. Superconductivityinduced anomalies in the spin excitation spectra of underdoped $\mathrm{YBa}_{2} \mathrm{Cu}_{3} \mathrm{O}_{6+\mathrm{x}}$, Phys. Rev. Lett. 78, 713-716 (1997).

[9] Dai, P., Yethiraj, M., Mook, H.A., Lindemer, T.B. \& Dogan, F. Magnetic dynamics in underdoped $\mathrm{YBa}_{2} \mathrm{Cu}_{3} \mathrm{O}_{7-\mathrm{x}}$ : Direct observation of a superconducting gap, Phys. Rev. Lett. 77, 5425- 5428 (1996).

[10] Bourges, P., et al. Shifting of the magnetic- resonance peak to lower energy in the superconducting state of underdoped $\mathrm{YBa}_{2} \mathrm{Cu}_{3} \mathrm{O}_{6.8}$, Europhysics Lett. 38, 313-318 (1997).

[11] Bulut, N. \& Scalapino, D.J. Neutron scattering from a collective spin fluctuation mode in a $\mathrm{CuO}_{2}$ bilayer, Phys. Rev. B53, 5149-5152 (1996). 
[12] Blumberg, G., Stojkovic, B.P. \& Klein, M.V. Antiferromagnetic excitations and van Hove singularities in $\mathrm{YBa}_{2} \mathrm{Cu}_{3} \mathrm{O}_{6+\mathrm{x}}$, Phys. Rev. B52, 15741-15744 (1995).

[13] Abrikosov, A.A. Neutron peak in the extended-saddle- point model of high-temperature superconductors, Phys. Rev. B57, 8656- 8661 (1998).

[14] Liu, D.Z., Zha, Y. \& Levin, K. Theory of Neutron Scattering in the Normal and Superconducting States of $\mathrm{YBa}_{2} \mathrm{Cu}_{3} \mathrm{O}_{6+\mathrm{x}}$, Phys. Rev. Lett. 75, 4130-4133 (1995).

[15] Mazin, I.I. \& Yakovenko, V.M. Neutron scattering and superconducting order parameter in $\mathrm{YBa}_{2} \mathrm{Cu}_{3} \mathrm{O}_{7}$, Phys. Rev. Lett. 75, 4134-4137 (1995).

[16] Onufrieva, F. \& Rossat-Mignod, J. Theory of spin dynamics in high- $T_{c}$ copper oxide superconductors: Application to neutron scattering, Phys. Rev. B52, 7572-7603 (1995).

[17] Onufrieva, F. Evidence for $d_{x^{2}-y^{2}}$ Symmetry of the Superconducting Order Parameter in YBCO from Neutron-Scattering Data, Physica C251, 348-354 (1995).

[18] Millis, A.J. \& Monien, H. Bilayer coupling in the yttrium- barium family of high-temperature superconductors, Phys. Rev. B54, 16172-16178 (1996).

[19] Morr, D.K. \& Pines, D. The resonance peak in cuprate superconductors, Phys. Rev. Lett. 81, 1086-1089 (1998).

[20] Yin, L., Chakravarty, S. \& Anderson, P.W. The neutron peak in the interlayer tunneling model of high temperature superconductors, Phys. Rev. Lett. 78, 3559-3562 (1997).

[21] Zhang, S.C. A unified theory based on $\mathrm{SO}(5)$ symmetry of superconductivity and antiferromagnetism, Science 275, 1089-1096 (1997).

[22] Demler, E., Kohno, H. \& Zhang, S.C. $\pi$-excitation of the t-J model, Phys. Rev. B58, 5719-5730 (1998).

[23] Scalapino, D.J. \& White, S.R. The Superconducting Condensation Energy and an Antiferromagnetic Exchange Based Pairing Mechanism, preprint cond-mat/9805075 at http://xxx.lanl.gov.

[24] Demler, E. \& Zhang, S.C. Quantitative Determination of a Microscopic Mechanism of High Tc Superconductivity, Nature 396, 733-735 (1998). 
[25] Norman, M.R. et al. Destruction of the Fermi surface underdoped high$\mathrm{T}_{\mathrm{c}}$ superconductors, Nature 392, 157-160 (1998).

[26] Shen, Z.X. et al. Temperature-induced momentum-dependent spectral weight transfer in $\mathrm{Bi}_{2} \mathrm{Sr}_{2} \mathrm{CaCu}_{2} \mathrm{O}_{8+\delta}$, Science 280, 259-262 (1998).

[27] Gu, G.D., Takamuku, K., Koshizuka, N. \& Tanaka, S. Large single crystal Bi-2212 along c-axis prepared by floating zone method, J. Crystal Growth 130, 325-329 (1998).

[28] Bourges, P. et al. High-energy spin excitations in $\mathrm{YBa}_{2} \mathrm{Cu}_{3} \mathrm{O}_{6.5}$, Phys. Rev. B56, R11439-R11442 (1997).

[29] Fong, H.F. et al. Effect of nonmagnetic impurities on the magnetic resonance peak in $\mathrm{YBa}_{2} \mathrm{Cu}_{3} \mathrm{O}_{7}$, Phys. Rev. Lett. (in press); also preprint cond-mat/9812047 at http://xxx.lanl.gov.

[30] Norman, M.R. \& Ding, H. Collective modes and the superconductingstate spectral function of $\mathrm{Bi}_{2} \mathrm{Sr}_{2} \mathrm{CaCu}_{2} \mathrm{O}_{8}$, Phys. Rev. B57 R11089R11092 (1998).

[31] Shen, Z.X. \& Schrieffer, J.R. Momentum, temperature, and doping dependence of photoemission lineshape and implications for the nature of the pairing potential in high- $\mathrm{T}_{\mathrm{c}}$ superconducting materials, Phys. Rev. Lett. 78, 1771-1774 (1997). 


\section{Figure Captions}

1. Constant-energy scans, at excitation energy $43 \mathrm{meV}$, of the neutron scattering intensity as a function of the wave vectors (a) $\mathbf{Q}_{\|}$and (b) $Q_{\perp}$ parallel and perpendicular to the $\mathrm{CuO}_{2}$ planes, respectively. The wave vector $\mathbf{Q}=(\mathrm{H}, \mathrm{K}, \mathrm{L})$ is given in reciprocal lattice units (r.l.u.), that is, in units of the reciprocal lattice vectors $a^{*} \sim b^{*}=1.64 \AA^{-1}$ and $c^{*}=0.20 \AA^{-1}$. In these units, $\mathbf{Q}_{\|}=(0.5,0.5)$ corresponds to the antiferromagnetic wave vector $(\pi / a, \pi / a)$. The points in (a) are raw data taken at temperatures $10 \mathrm{~K}\left(<\mathrm{T}_{\mathrm{c}}\right)$ and $100 \mathrm{~K}\left(>\mathrm{T}_{\mathrm{c}}\right)$. In (b), the intensity at $100 \mathrm{~K}$ was subtracted from that at $10 \mathrm{~K}$. The line in (a) is the result of a fit to a Gaussian profile while that in (b) is the function $\sin ^{2}\left(\pi z_{\mathrm{Cu}} L\right)$, where $z_{\mathrm{Cu}}=0.109$ is the distance between nearest-neighbor copper atoms in the $\hat{c}$-direction perperdicular to the planes, expressed as a fraction of the lattice parameter $c=30.8 \AA$. This modulation is due to magnetic interactions between adjacent $\mathrm{CuO}_{2}$ layers and has the same periodicity and phase as in $\mathrm{YBa}_{2} \mathrm{Cu}_{3} \mathrm{O}_{7}$. The bars represent the experimental wave vector resolution (full width at half maximum).

2. Difference spectrum of the neutron intensities at $\mathrm{T}=10 \mathrm{~K}\left(<\mathrm{T}_{\mathrm{c}}\right)$ and $\mathrm{T}=100 \mathrm{~K}\left(>\mathrm{T}_{\mathrm{c}}\right)$, at wave vector $\mathrm{Q}=(0.5,0.5,-14)$. The bar respresents the instrumental energy resolution, the line is a guide-to-the-eye.

3. Temperature dependence of the neutron intensity at energy $43 \mathrm{meV}$ and wave vector $\mathbf{Q}=(0.5,0.5,-14)$. The intensity falls to background level above $T_{c}=91 \mathrm{~K}$ (Fig. 1). The line is a guide-to-the-eye. 

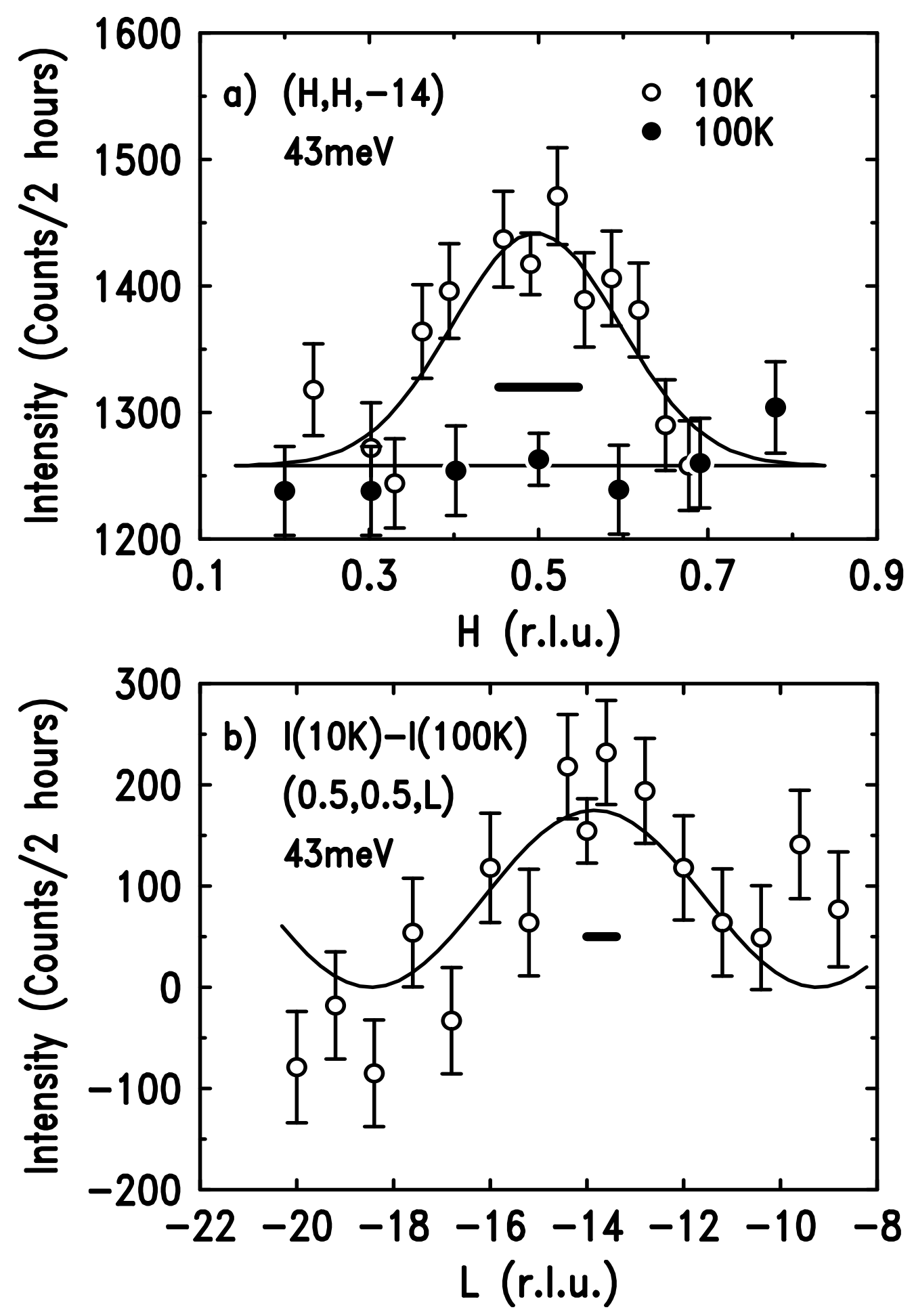


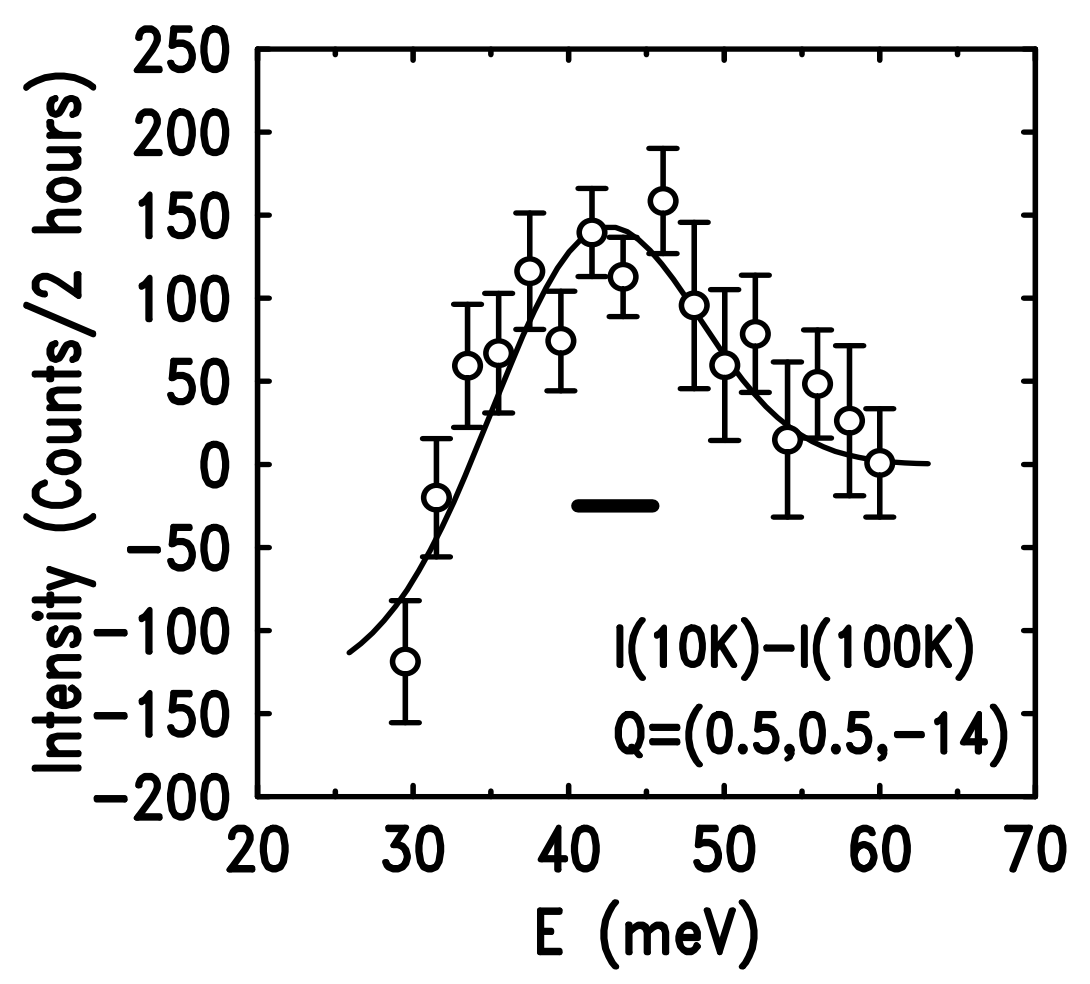




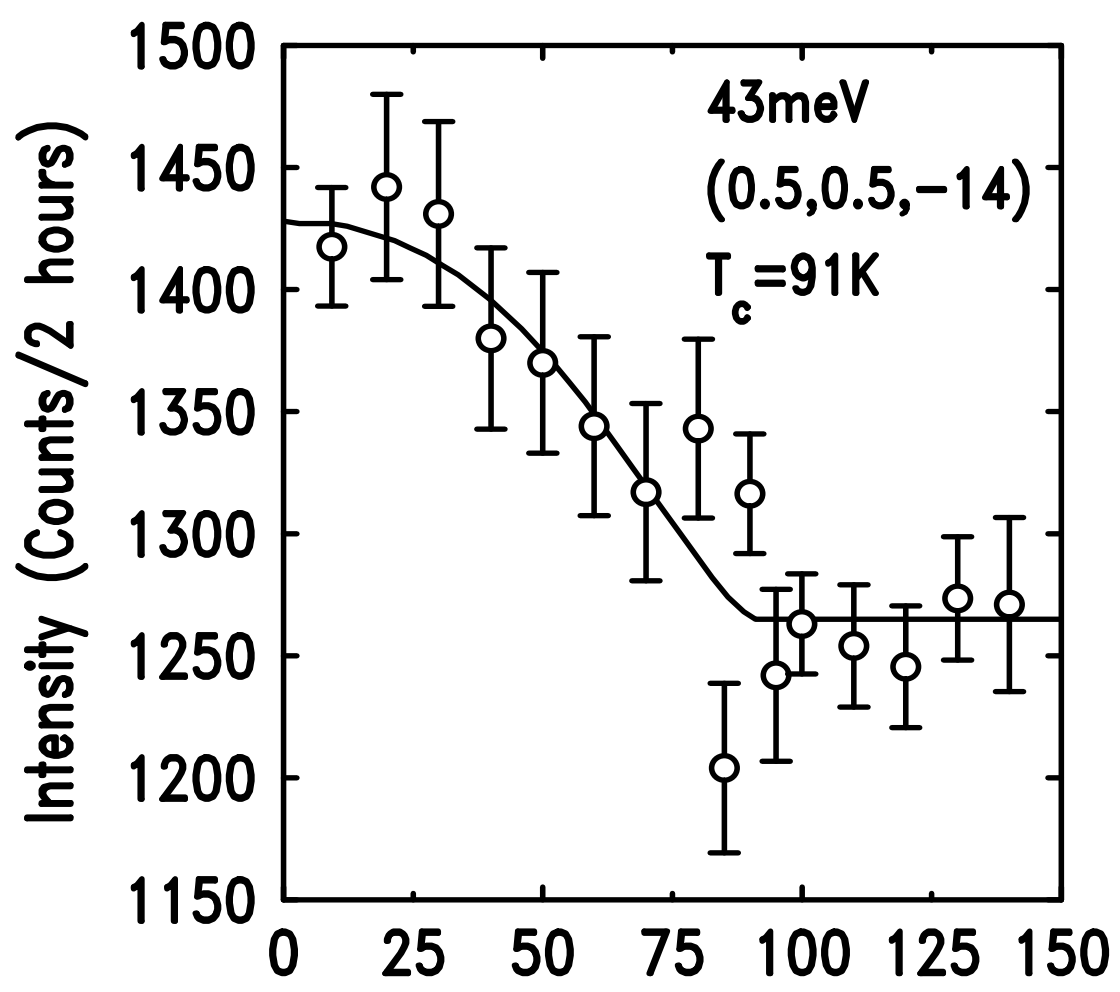

